\title{
INSTRUMENTOS DE LA POLITICA DE ORDENAMIENTO AMBIENTAL DEL TERRITORIO. UN ESTUDIO DEL MARCO NORMATIUO EN LA ARGENTINA CONTEMPORÁNEA. PI 18CQQ4 SGCYT UNNE
}

\author{
ROMAGNOLI, Venettia y FOGAR, Sandra \\ venettiaromagnoli@hotmail.com \\ Dra. Arq. Secretaría de Investigación de la FAU-UNNE, profesora adjunta \\ de TFC-TVB, FAU-UNNE. Investigadora adjunta del CONICET. Integrante \\ del Instituto de Investigación y Desarrollo en Vivienda, IIDVI (FAU- \\ UNNE), y del Instituto de Investigación para el Desarrollo Territorial \\ y del Hábitat Humano, IIDTHH (UNNE-CONICET). Codirectora de la \\ Maestría en Gestión Ambiental del Territorio Urbano (MGATU).
}

Mg. Arq. Jefa de Trabajos Prácticos con dedicación exclusiva de TFC-TVA, FAUUNNE. Directora de la Maestría Gestión Ambiental del Territorio Urbano (MGATU).

\section{PALABRAS CLAUE}

Políticas de ordenamiento ambiental del territorio; investigación; formación de posgrado.

\section{RESUMEN}

El propósito del presente trabajo consiste en difundir los primeros avances del proyecto de investigación denominado "Instrumentos de la política de ordenamiento ambiental del territorio. Un estudio del marco normativo en la Argentina contemporánea", acreditado por la Secretaría General de Ciencia y Técnica de la UNNE.

Se asume la perspectiva crítica del conocimiento, y con ello la construcción de saberes en el marco de acciones de vinculación, mediadas por la articulación de actividades de investigación con actividades de formación de posgrado, perteneciente al ámbito de la Maestría de Gestión Ambiental del Territorio Urbano, que se dicta en la FAU-UNNE.

\section{KEYWORDS}

environmental management policies of the territory - research - postgraduate training.

ABSTRACT

The aim of this work is to spread the process of formulation and objectives, as well as the first advances of a research project accredited by the General Secretary of Science and Technology of the UNNE, called "Instruments of the territory's environmental management policy. A study of the regulatory framework in contemporary Argentina", that aims to generate an advance in the production of knowledge and initiate linkage and feedback actions between postgraduate training and research activities, proposing as a specific place of work, to the MGATU, within the Faculty of Architecture and Urbanism of the UNNE. 


\section{OBJETIUOS}

\section{Objetivo general}

- Identificar y analizar los instrumentos normativos de la política de ordenamiento ambiental del territorio en la Argentina contemporánea, específicamente los correspondientes a la región del NEA.

\section{Objetivo específicos}

- Construir un corpus teórico y empírico que permita el desarrollo de un marco interpretativo propio para la sistematización y análisis de los instrumentos normativos de la política de ordenamiento ambiental de la Argentina contemporánea.

- Diseñar y elaborar un inventario tipológico digitalizado integral del marco normativo de la política de ordenamiento ambiental del territorio de la Argentina contemporánea.

- Contrastar a la luz del marco interpretativo propio los instrumentos normativos que conforman el inventario.

- Desarrollar pautas y lineamientos para contribuir a la consolidación de un marco normativo coherente y unificado que permita su integración efectiva en el sistema de planificación integrado por el plan, los instrumentos normativos y la gestión.

\section{INTRODUCCIÓN O PLANTEO DEL PROBLEMA}

La formulación y desarrollo del proyecto de investigación denominado "Instrumentos de la política de ordenamiento ambiental del territorio. Un estudio del marco normativo en la Argentina contemporánea" (PI 18C004 SGCyT, UNNE) persigue una doble finalidad. Por un lado, se pretende aportar a un espacio de vacancia en el campo de los estudios de políticas de ordenamiento ambiental del territorio que han tomado como objeto el caso de Argentina y de las provincias de la región NEA en particular. Por otro lado, coherentemente con las nuevas políticas institucionales de nivel regional, nacional y de la propia UNNE, se aspira a desarrollar acciones de vinculación y retroalimentación entre actividades de formación de posgrado y actividades de investigación. En tal sentido, resulta oportuna la designación de la Maestría en Gestión Ambiental del Territorio Urbano (MGATU) que se dicta en la Facultad de Arquitectura y Urbanismo de la UNNE como ámbito de trabajo apropiado para el proceso de formación que involucra este proyecto de investigación.

\footnotetext{
1. Integrantes del proyecto: Dra. Arq. Venettia Romagnoli (directora); Dr. Arq. Daniel Bedrán (investigador) Mgter. Arq. Sandra Fogar (investigadora); Mgter. Linda Peso (investigadora); Arq. Carlos Roces (investigador); Lic. María Emilia Fernández (investigadora); los tesistas de posgrado: José Luis Alunni; Gabriela Barberán; Carolina Bernasconi; Juana Bordón; Javier Bozzolo; Noemí Cabali; Miriam Carballo; Diego Casas; Diego Fernández; Marili Garrafa; Gerardo Giménez; Sabrina Greve; Miriam Passi Pérez Daniela Petrucci y Gabriel Roggero y la becaria de investigación: Lucrecia Seluy.
}

La construcción del proyecto de investigación al que nos referimos, que se inicia a principios del año 2018, es el producto del trabajo colectivo de un equipo integrado por dos miembros de la dirección de la MGATU, seis docentes investigadores, una becaria de investigación y quince maestrandos ${ }^{7}$ tesistas de la MGATU (que provienen de diversos campos disciplinares -arquitectura, ingeniería, abogacía, geografía, entre otros-). Con respecto a estos últimos, se aspira a que esta tarea aporte además al desarrollo de las tesis en curso.

Es así que, con la formulación y desarrollo de este proyecto, se pretende ocupar espacios de construcción y transferencia de conocimientos mediante dos estrategias de trabajo: una a través de la articulación entre actividades de formación de posgrado -que posibiliten la constitución de un espacio para desarrollo de las tesis de los maestrandos y tareas de investigación-; otra, vinculada con la temática relativa al estudio de políticas de ordenamiento ambiental del territorio, circunscriptas al ámbito nacional y regional.

La perspectiva desde la cual se formula el proyecto concibe las políticas de ordenamiento ambiental del territorio como un sistema dinámico constituido por tres elementos estructurales que se interrelacionan e interdefinen: el plan territorial con sus instrumentos operativos (programas y proyectos), el marco normativo que garantice su consolidación como política de Estado-que trascienda situaciones coyunturales-yel modelo de gestión (cuya estructura organizacional y prácticas administrativas permitan 
su aplicación en el territorio). En primera instancia, la inexistencia, debilidad o incongruencia de algunos de estos tres componentes condiciona fuertemente el cumplimiento de los objetivos que generalmente persiguen las políticas públicas, tendientes a asegurar el equilibrio territorial para garantizar un desarrollo socioeconómico equitativo que preserve y proteja el ambiente, con el fin de mejorar la calidad de vida de la población; y en segundo lugar, limitan el rol protagónico que debe asumir el Estado en esta materia.

Estudios previos (Erbiti, 2011; Barreto, 2015, Romagnoli, 2018, Seluy y Romagnoli, 2018, entre otros) han señalado que el marco normativo vigente en Argentina en materia de ordenamiento ambiental del territorio presenta problemas vinculados con la débil consolidación, fragmentación, dispersión, superposición y vacancia. La investigación parte del supuesto de que el marco normativo de la política de ordenamiento ambiental del territorio en la Argentina contemporánea, y particularmente en las provincias del NEA, presenta los problemas señalados, lo cual limita la capacidad de actuación política, concebida como sistema integrado por los tres elementos estructurales (plan, instrumentos normativos y gestión). Desde esta perspectiva, el marco normativo de la política de ordenamiento ambiental del territorio se constituye en objeto de estudio central para analizar problemáticas del sistema de planificación vigente en Argentina y específicamente en la región NEA, y contribuir ulteriormente al campo del ordenamiento ambiental del territorio.

\section{DESARROLLO O RESULTADOS}

Las grandes transformaciones en el territorio de las ciudades estuvieron básicamente circunscriptas a cuatro fenómenos: el impacto directo e indirecto de un modelo económico, el crecimiento demográfico, los cambios producidos por los avances científico-tecnológicos y las políticas y gestión de los estados. Aunque en general los cambios siempre quedaron sujetos a procesos que más tarde o más temprano se internacionalizaron, las grandes transformaciones urbanas en particular inician también un ininterrumpido proceso de mutaciones que determinaron modos particulares de ocupación del territorio. Esas mutaciones se dieron -con rasgos diferenciados pero sostenidamente-, desde el barroco y el clasicismo hasta la actual era de la globalización, y que a partir de fines del siglo $X X$, adquieren una inusitada dinámica.

En un escenario de profundos cambios geopolíticos, atravesado por la revolución de las comunicaciones, la ocupación del territorio exige además analizar a la luz de un posicionamiento crítico herramientas de gestión territorial, que posibiliten contribuir a la consolidación de un marco normativo coherente y unificado, que admita la integración efectiva de sus componentes, vistos como sistema de planificación integrado. Por ello, esta investigación se propone analizar a la luz de un marco teórico crítico los instrumentos normativos de la política de ordenamiento ambiental del territorio en la Argentina, y particularmente en la región del
NEA, en virtud de contribuir a la consolidación de un marco normativo coherente y unificado, que permita su integración efectiva al sistema complejo de plan, instrumentos normativos y gestión.

Tanto la instancia de formulación del proyecto como la actual de desarrollo han constituido experiencias de trabajo colectivo, con logros alcanzados en diferentes espacios de actuación. La diversidad de los actores que conforman el equipo, en cuanto a ámbitos de desempeño profesional y laboral, edades y formación de grado, ha exigido a la directora del proyecto-que es quien conduce las tareas-realizar modificaciones en la planificación. Así, la actividad del primer año de trabajo está orientada a la construcción de un corpus teórico y empírico, lo cual posibilitará sistematización y análisis de instrumentos normativos actuales de la política de ordenamiento ambiental de la Argentina. Esta modificación en la organización se apoya en el hecho de que el grupo de maestrandos no cuenta con experiencias previas de participación en proyectos de investigación, lo cual requirió pensar en instancias de revisión del estado del debate teórico, para ampliación y profundización del marco conceptual sobre la problemática y elaboración de documento interno que contenga el marco teórico provisorio construido mediante la revisión teórica. Los cambios introducidos, si bien han requerido redoblados esfuerzos de reprogramación, no fueron obstáculo alguno en el desarrollo de las actividades, pues posibilitaron enriquecer la propuesta a partir de la participación y el aporte de todos los miembros del equipo. 


\begin{tabular}{|c|c|c|}
\hline CUF & \multicolumn{2}{|c|}{ Síntesis de la metodología de la investigación } \\
\hline OBJE & ACTIVIDADES & MÉTO \\
\hline \multirow{2}{*}{$\begin{array}{l}\text { Construir un corpus } \\
\text { teórico y empírico que } \\
\text { permita el desarrollo de } \\
\text { un marco interpretativo } \\
\text { propio para la sistema- } \\
\text { tización y análisis de los } \\
\text { instrumentos norma- } \\
\text { tivos de la política de } \\
\text { ordenamiento ambiental } \\
\text { de la Argentina contem- } \\
\text { poránea. }\end{array}$} & $\begin{array}{l}\text { Revisión del estado del deba- } \\
\text { te teórico para la ampliación } \\
\text { y profundización de un marco } \\
\text { conceptual sobre la proble- } \\
\text { mática. }\end{array}$ & \multirow{2}{*}{$\begin{array}{l}\text { Tareas de gabinete. } \\
\text { Revisión crítica de bibliografía y antecedentes con el objeto de } \\
\text { construir el marco teórico a la luz del cual se analizará el objeto } \\
\text { de estudio. } \\
\text { Revisión del diseño del plan de relevamiento y análisis. } \\
\text { Lectura orientada al intercambio entre integrantes del equipo de } \\
\text { investigación, con el objeto de sistematizar resultados y produc- } \\
\text { ciones propias. } \\
\text { Se prevé la participación de los miembros del grupo en congresos, } \\
\text { seminarios, encuentros que permitan discutir en ámbitos más } \\
\text { amplios el marco teórico provisorio. }\end{array}$} \\
\hline & $\begin{array}{l}\text { Elaboración de un documento } \\
\text { interno con el marco teórico } \\
\text { provisorio construido me- } \\
\text { diante la revisión teórica. }\end{array}$ & \\
\hline \multirow{6}{*}{$\begin{array}{l}\text { Diseñar y elaborar un } \\
\text { inventario tipológico } \\
\text { digitalizado integral } \\
\text { del marco normativo } \\
\text { de la política de } \\
\text { ordenamiento } \\
\text { ambiental del territorio } \\
\text { de la Argentina } \\
\text { contemporánea. }\end{array}$} & $\begin{array}{l}\text { Diseño del plan de releva- } \\
\text { miento. }\end{array}$ & \multirow{6}{*}{$\begin{array}{l}\text { Instancia de Diseño Empírico (construcción de datos). } \\
\text { Instancia Operativa (construcción de la base empírica), para lo cual } \\
\text { se desarrollarán tareas de gabinete y de campo. Recolección de } \\
\text { la información secundaria: } \\
\text { - Preparación y ejecución del trabajo de campo; producción de } \\
\text { información primaria: detección de informantes clave, entrevis- } \\
\text { tas, etc. } \\
\text { - Sistematización y ordenamiento de la información. } \\
\text { - Recolección y sistematización de información secundaria. } \\
\text { Se prevé la conformación de comisiones de trabajo con instancias } \\
\text { de puesta en común, discusión y debates. }\end{array}$} \\
\hline & $\begin{array}{l}\text { Construcción de un glosario } \\
\text { sobre la base de definiciones } \\
\text { oficiales y operativas. }\end{array}$ & \\
\hline & $\begin{array}{l}\text { Diseño del modelo de inven- } \\
\text { tario: dimensiones, variables } \\
\text { e indicadores. }\end{array}$ & \\
\hline & $\begin{array}{l}\text { Relevamiento de la informa- } \\
\text { ción. }\end{array}$ & \\
\hline & $\begin{array}{l}\text { Carga de la información al } \\
\text { soporte digital diseñado. }\end{array}$ & \\
\hline & & \\
\hline \multirow[b]{3}{*}{$\begin{array}{l}\text { Contrastar a la luz del } \\
\text { marco interpretativo } \\
\text { los instrumentos } \\
\text { normativos que } \\
\text { conforman } \\
\text { el inventario. }\end{array}$} & Diseño del & \multirow[b]{3}{*}{$\begin{array}{l}\text { Análisis e interpretación de datos a la luz del marco interpretativo. } \\
\text { Se prevén: } \\
\text { - Instancias de trabajo que posibiliten la construcción colectiva } \\
\text { en un ámbito de trabajo multidisciplinar. } \\
\text { - Producción de ponencias y artículos que muestren avances de } \\
\text { la metodología de análisis de los instrumentos normativos de la } \\
\text { política de ordenamiento ambiental del territorio en Argentina y } \\
\text { particularmente en las provincias del NEA y permitan la revisión } \\
\text { del conocimiento construido. }\end{array}$} \\
\hline & Desa & \\
\hline & $\begin{array}{l}\text { Elaboración de un documento } \\
\text { interno con los resultados del } \\
\text { análisis. }\end{array}$ & \\
\hline \multirow{2}{*}{$\begin{array}{l}\text { Desarrollar pautas y li- } \\
\text { neamientos para contri- } \\
\text { buir a la consolidación } \\
\text { de un marco normativo } \\
\text { coherente y unificado que } \\
\text { permita su integración } \\
\text { efectiva al sistema de pla- } \\
\text { nificación integrado por } \\
\text { el plan, los instrumentos } \\
\text { normativos y la gestión }\end{array}$} & $\begin{array}{l}\text { Formulación de propuestas } \\
\text { de intervención. }\end{array}$ & \multirow{2}{*}{$\begin{array}{l}\text { Tareas de gabinete. } \\
\text { Técnicas de formulación de propuestas, que integren el marco } \\
\text { interpretativo construido con los resultados del análisis de los } \\
\text { instrumentos normativos. Sistematización de los resultados. } \\
\text { Elaboración de documento e informes finales. } \\
\text { Firma de actas de transferencia de resultados del proyecto, vía } \\
\text { articulación con organismos gubernamentales. }\end{array}$} \\
\hline & $\begin{array}{l}\text { Elaboración de documen- } \\
\text { tos de transferencia/exten- } \\
\text { sión según condiciones de } \\
\text { transferencia y agentes que } \\
\text { transferir. }\end{array}$ & \\
\hline
\end{tabular}


En cuanto a la metodología empleada en la investigación, se corresponde con un modelo inductivo-cualitativo; consecuentemente el diseño metodológico requerirá-al no encuadrarse en una teoría sustantiva- una estrategia también inductiva, en la cual se pretende que el marco interpretativo que se construya se constituya en el marco conceptual desde el cual se elaboren las categorías de análisis, que se articularán con los datos que se vayan obteniendo durante el desarrollo del trabajo empírico. El siguiente cuadro muestra la síntesis de objetivos, actividades, métodos y técnicas propuestos (cuadro1).

Con respecto al estado de avance del plan de trabajo, la instancia actual de construcción del marco interpretativo ha posibilitado reflexionar acerca de algunos núcleos conceptuales que -si bien aún se consideran provisorios- permitieron clarificar nociones, sobre la base de un trabajo deconsenso y construcción colectiva, que son centrales para comprender el objeto de estudio. En relación con ello, se explicitan posicionamientos relativos a los núcleos conceptuales de la investigación.

\section{Sobre la concepción de territorio}

El territorio es concebido como espacio natural transformado por la acción de todos, para la utilización de sus recursos, que se caracteriza por tener una capacidad limitada de acogida, y que experimenta sucesivas y constantes transformaciones. Estas características lo constituyen en escenario de fenómenos multidimensionales, complejos y dinámicos (Kullock, 2010), y en espacio de pujas de intereses de diferentes actores,

\section{FIGURA 1 Ciclo de las políticas públicas}

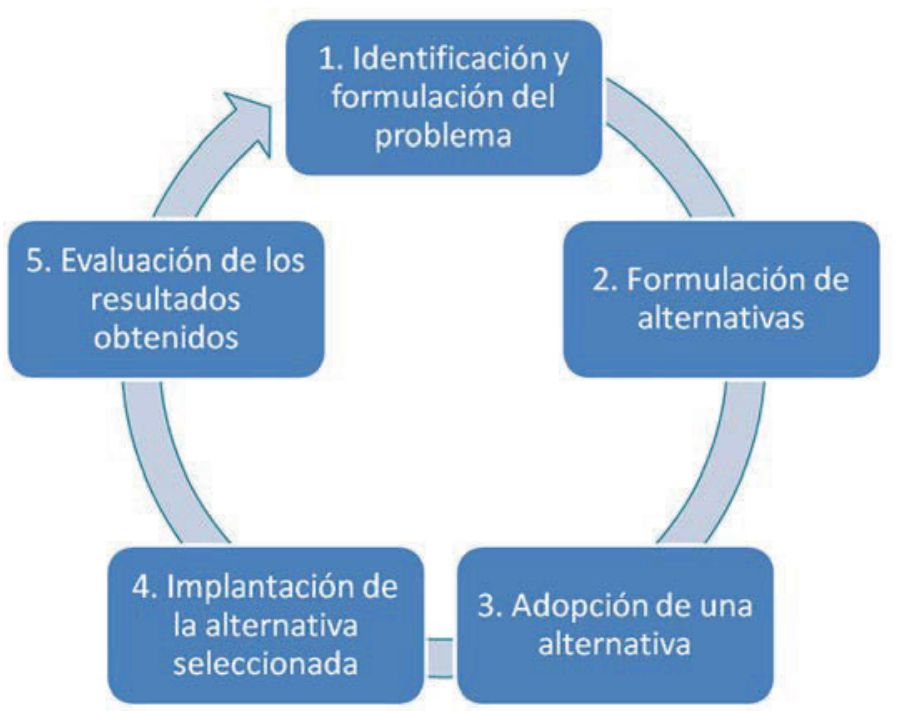

Fuente: Romagnoli, Venettia (2015), sobre la base de Subirats(1989) y Tamayo Sáez (1997)

con asimétricas cuotas de poder, que ponen en juego las estrategias con las que cuentan para apropiarse de sus recursos e incidir en su configuración (Romagnoli, 2015 y 2017).

\section{Sobre la concepción que guía el proceso de construcción de políticas territoriales}

Las políticas públicas de ordenamiento territorial son analizadas en este trabajo desde una perspectiva que intenta superar enfoques tecnocráticos, y son concebidas como producto de disputas entrelos diversos actores interesados e involucrados en las distintas instancias implicadas en su configuración (Subirats, 1989; Tamayo Sáez, 1997; Jobert, 2005; Bourdieu, 2001 y Romagnoli, 2015 y 2017).

Se reconoce el carácter cíclico o procesual de las políticas públicas
(Subirats, 1989 y Tamayo Sáez, 1997). Desde esta perspectiva, el proceso seguido por toda política pública se inicia cuando un determinado gobierno asume y define la existencia de un problema para proponer una solución, analiza alternativas en función de los intereses implicados y los recursos disponibles, se fija determinados objetivos y toma decisiones que desencadenan la implementación de acciones para obtener ciertos resultados. Cada una de las fases, instancias o momentos del "ciclo de la políticas públicas" se encuentra atravesada y determinada por la lucha y el conflicto, y se constituye en el resultado del triunfo o hegemonía de un grupo social sobre otro (figura 1).

En trabajos previos (Romagnoli, 2015,2016 y 2017) se señala que el ciclo de las políticas públicas debe ser analizado desde la perspectiva 
de las necesidades (Fraser, 1986 y 1991), lo que implica entender que las necesidades que fundamentan las políticas públicas no están "simplemente dadas", sino que son "construidas" a partir de procesos de luchas políticas. En tales procesos, es posible además identificar distintos momentos: un primer momento de lucha por establecer el estatuto político de una determinada necesidad, con o sin alcanzar legitimidad política; un segundo momento de lucha por la interpretación de esa necesidad, en el cual se "juega el poder" para definirla de manera hegemónica y de esta manera determinar la forma "conveniente" de satisfacerla, y finalmente, un tercer momento nos muestra el debate sobre la satisfacción en el plano de los recursos, provisiones y administración de la respuesta política (figura 2 ).

\section{Sobre la concepción de las políticas territoriales en articulación con los actores involucrados}

En relación con los diferentes tipos de actores que participan del proceso, algunos autores como Romagnoli, Venettia (2015 y 2016) plantean la necesidad de distinguirlos y tipificarlos en función de los intereses que representan y los discursos de los cuales seapropian. Desde esta mirada, se identifican al menos tres tipos de actores: estatales, económicos y sociales, que polemizan y entran en disputa en distintos momentos e instancias del proceso de construcción de las políticas territoriales. Pero, a su vez, los actores se agrupan en dos grandes categorías:

a) los tradicionalmente subordinados, que pueden distinguirse entre

- actores sociales (demandantes, destinatarios de las políticas, organizaciones sociales);

- actores académicos (expertos y científicos sociales posicionados desde una perspectiva crítica), que se caracterizan por desplegar y diseñar estrategias de politización de necesidades (demandas) o interpretaciones contrapuestas a las

\section{FIGURA 2}

El proceso de configuración de las necesidades en las políticas públicas

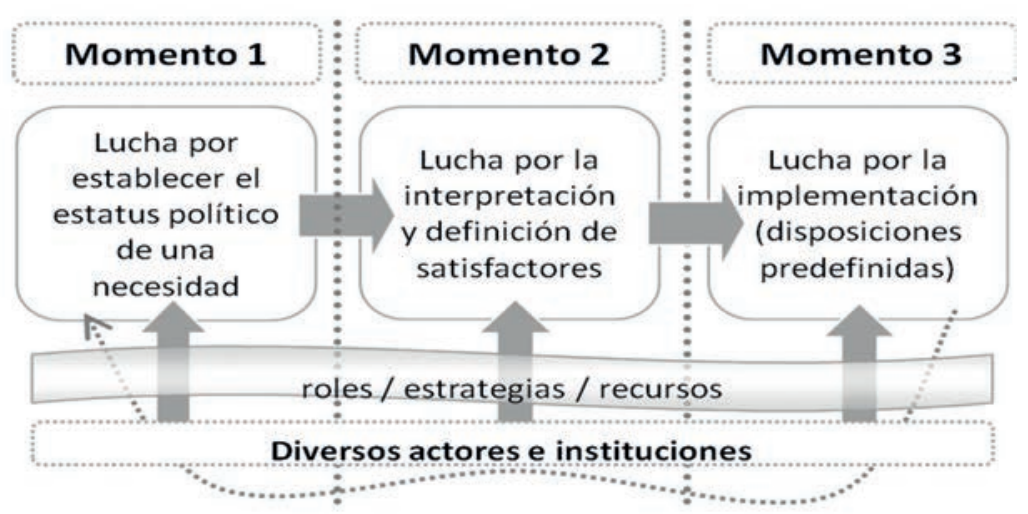

Fuente: Romagnoli, Venettia (2015), sobre la base de Fraser (1986 y 1991) hegemónicas a partir de recursos políticos (manifestaciones, marchas, etc.);

b) los tradicionalmente hegemónicos, vinculados con el mercado, y que a través de los recursos económicos pueden ejercer presiones manifiestas u ocultas, con el fin de que las políticas respondan a sus intereses;

c) los actores estatales, que se desempeñan en los distintos niveles jurisdiccionales implicados, y que siendo portadores de los "recursos normativos", se encargan de la traducción de las necesidades

\section{Tipos de actores y discursos involucrados \\ FIGURA 3 Tipos de actores y discursos invol las
en el proceso de configuración de las necesidades en las políticas públicas}

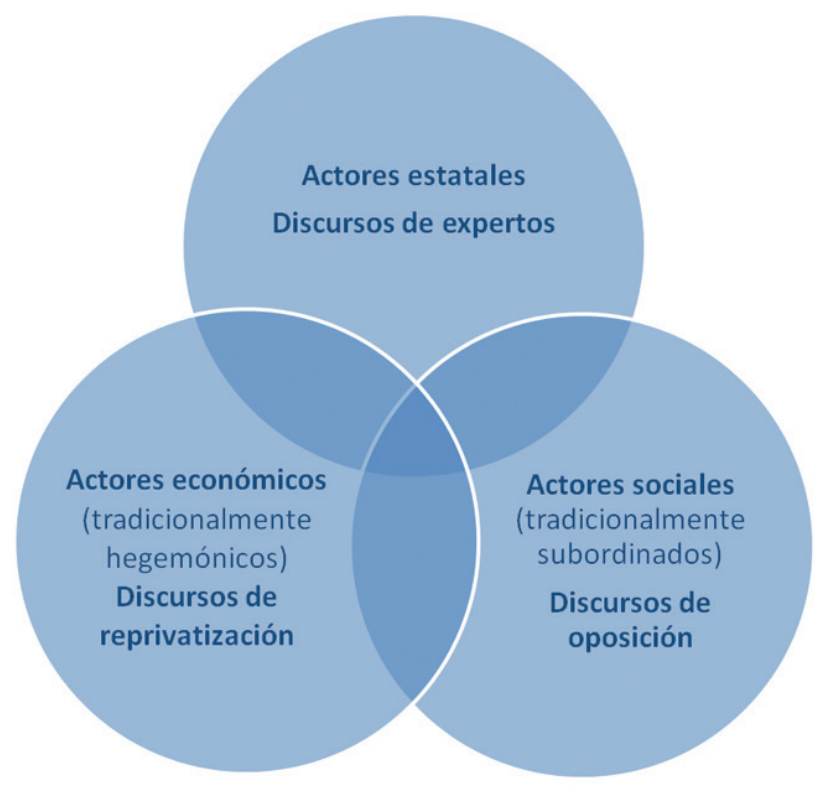

Figura 3. Fuente. Romagnoli, Venettia (2015), sobre la base de Fraser (1986) 
politizadas a "necesidades administrables", recurriendo a los discursos de sistemas funcionalistas de planificadores, expertos en políticas y científicos sociales de la corriente dominante (Fraser, 1986) (figura 3).

Todos ellos operan paralelamente poniendo en juego intereses individuales y colectivos que inciden en la configuración (de manera directa e indirecta) de las políticas territoriales.

\section{Sobre el rol del Estado en la concepción de las políticas territoriales}

En el complejo entramado de actores que intervienen en este campo, el Estado asume un rol en función de su ethos social dominante. Vinculado con ello, Esping Anderesen (1989) identifica tres modelos de Estado de Bienestar: el liberal, el corporativista y el socialdemócrata. Pero estos modelos están estrechamente vinculados con las políticas, a través de las cuales se plasma uno $u$ otro, y que obedecen al entramado de actores e intereses que acceden transitoriamente al poder y ejercen el gobierno de cada Estado, asignándoles características particulares a las políticas

Para Doyal y Gough (1994), las políticas públicas varían de acuerdo con los diferentes modelos de desarrollo, y por lo mismo no es posible sostener que una política de ordenamiento territorial sea neutral con respecto al modelo de desarrollo de la sociedad. Por el contrario, es constitutiva del conjunto de políticas sociales implementadas por un gobierno, que comprenden muchos otros aspectos, y su contenido y relaciones se encuentran siempre condicionados por la orien-

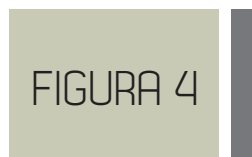

Relación entre las políticas de ordenamiento territorial y el modelo de desarrollo

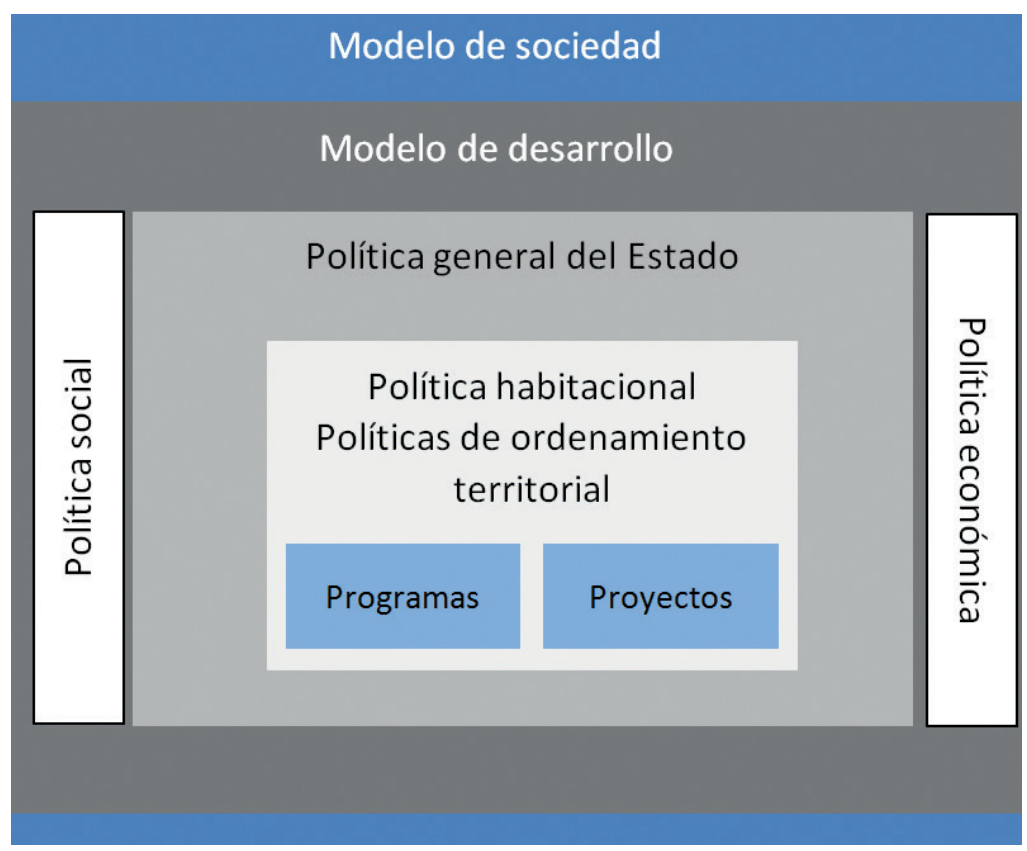

Fuente: Romagnoli, Venettia (2018), sobre la base de Barreto y otros (2014) y Romagnoli (2015)

tación del modelo de desarrollo impulsado por cada gobierno, y en consecuencia por el modelo de sociedad al que este aspira. Desde esta perspectiva, la comprensión de los problemas territoriales y las definiciones de su abordaje desde una política de ordenamiento territorial son indisociables de la orientación del modelo de desarrollo y de sociedad que promueven los distintos gobiernos (Barreto y otros, 2010, y Romagnoli, 2015) (figura 4).

\section{Sobre la concepción de las políticas territoriales}

En estrecha relación con lo planteado en el punto anterior, entendemos la política de ordenamiento territorial como la intervención directa de organismos del Estado de distintas jurisdicciones, realizada desde determinadas orientaciones políticas, sobre la organización y producción territorial, y que debe tener como objetivo asegurar el equilibrio territorial garantizando un desarrollo socioeconómico equitativo, protegiendo y preservando el ambiente con el fin de mejorar la calidad de vida de su población (Galarza Lucich, 2002, y Barreto, Ebel y Abildgaard, 2015).

Se entiende además que una política de ordenamiento territorial debe ser concebida como sistema integrado por tres instrumentos estructurales, que se interrelacionan e interdefinen: 
el plan territorial con sus instrumentos operativos (programas y proyectos), el marco normativo que garantice su consolidación como política de Estado que trascienda instancias coyunturales de gobiernos, y un modelo de gestión (estructura organizacional y prácticas administrativas que permitan su concreción en el territorio). La inexistencia, debilidad o incongruencia de algunos de estos tres elementos condiciona fuertemente el cumplimiento de los objetivos que se pretendan alcanzar y limita el rol protagónico que debe asumir el Estado para garantizarlos (figura 5).

El objeto de estudio de la presente investigación no ha sido abordado ni analizado desde la perspectiva que aquí se propone, por lo que consideramos que este trabajo será un aporte al conocimiento de un área que se considera de vacancia. El punto de partida de la perspectiva aquí asumida surge de recuperar los posicionamientos $^{2}$ de quienes han contribuido al estudio de las políticas públicas, de entre los cuales se destacan, a nivel internacional, los aportes de Bourdieu (2001), Tamayo Sáez (1997), Subirats (1989), Jones (1984) y Jobert (2005), quienes conciben las políticas públicas como productos emergentes de un ciclo o proceso caracterizado por la pugna y negociación de actores colectivos dentro del aparato estatal. Con respecto al ámbito de las teorías de las necesidades humanas, que entienden las políticas públicas como resultantes de procesos de configuración atravesados por la

\section{FIGURA 5.}

\section{Instrumentos de una política} de ordenamiento territorial

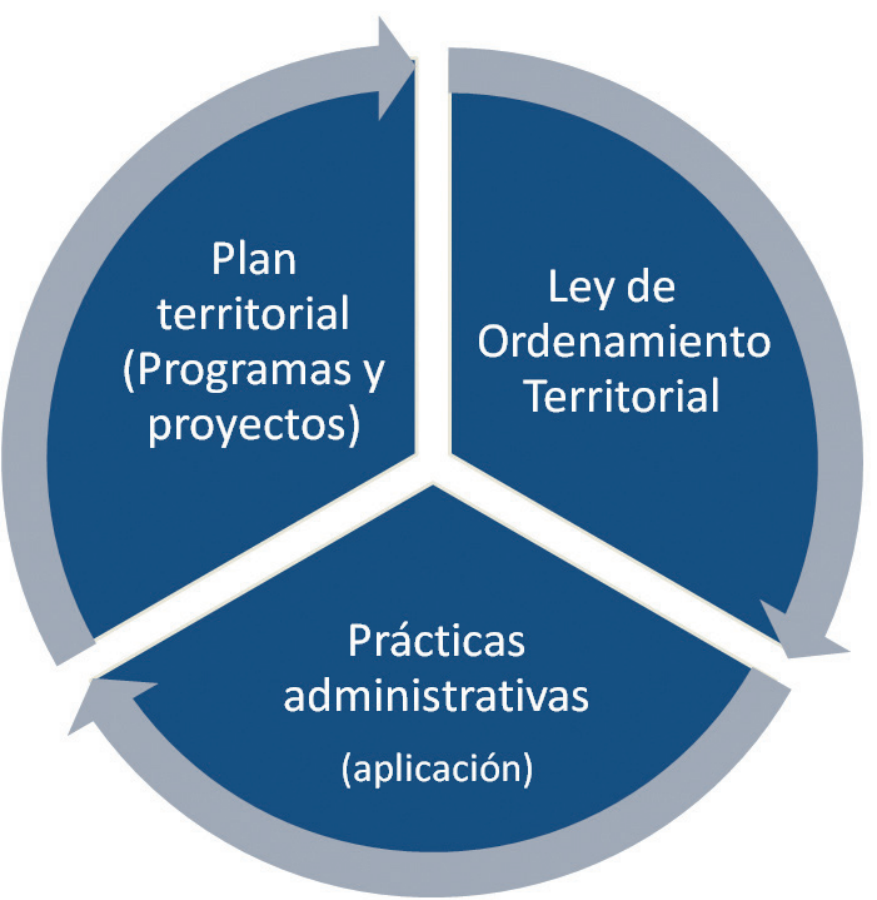

Figura 5. Fuente: Romagnoli, Venettia (2015), sobre la base de Galarza Lucich (2002) y Barreto y otros (2017)

disputa entre diversos actores, se recuperan los aportes teóricos de Nancy Fraser (1986 y 1991), a la luz de los cuales se analizan los momentos de dicho proceso y los tipos de actores involucrados. Desde esta misma mirada, que intenta superar posturas tecnocráticas acerca de las concepciones que guían las políticas públicas, los trabajos de Esping Anderesen (1989) y Doyal y Gough (1994) cobran relevancia al sostener su interdependencia con un modelo de desarrollo.

A nivel nacional y local, se consideran fundamentales los aportes de investigaciones llevadas adelante principalmente por el GID IIDVi (Barreto y otros, 2014; Romagnoli, 2015, 2016 y 2017; Benítez y Romagnoli,

2. Tal como se señaló en el apartado correspondiente a la Fundamentación en la descripción del proyecto, que se consigna en la presentación ante la SGCYT de la UNNE.

2017), del cual forman parte dos integrantes de este proyecto. Sibien sus indagaciones se circunscriben al ámbito de las políticas habitacionales, son relevantes el marco teórico y el enfoque metodológico asumidos, que se recuperan en el análisis de las políticas públicas de este trabajo.

En el campo específico de políticas de ordenamiento ambiental del territorio, interesan los trabajos de autores que provienen tanto del ámbito internacional como local. Es el caso de Galarza Lucich (2002), David Kullock (2010) y Barreto, Ebel y Abildgaard (2015), entre otros, que entienden la política de ordenamiento territorial como las intervenciones directas de organismos del Estado de distintas jurisdicciones, realizada desde determinadas orientaciones políticas, sobre la organización y producción territorial, y cuyo objetivo es asegurar 
el equilibrio territorial garantizando un desarrollo socioeconómico equitativo, protegiendo y preservando el ambiente, con el fin de mejorar la calidad de vida de su población.

En la misma dirección que Esping Anderesen (1989) y Doyal y Gough (1994), pero en relación específica con las políticas de ordenamiento ambiental del territorio, los estudios realizados por Barreto y otros (2010) y Romagnoli (2015) proponen un enfoque que sostiene que la política de ordenamiento territorial no es neutral respecto del modelo de desarrollo de la sociedad.

En relación con el recorte del objeto de estudio en este proyecto, se asume a partir de Barreto y otros (2017) y Romagnoli (2018) que una política de ordenamiento territorial debe mirarse desde la noción que la concibe como sistema complejo en el cual intervienen otros componentes, que están interrelacionados y son interdependientes. Este rasgo le confiere carácter de sistema integrado, que además involucra múltiples dimensiones a partir de las cuales estudiar este campo.

El sistema integrado, compuesto por elementos que son nucleares para el abordaje de políticas territoriales, son los tres ya mencionados en el punto anterior Sobre la concepción de las políticas territoriales: el plan, el marco normativo y el modelo de gestión. Su centralidad en el diseño de una política de ordenamiento se corresponde con las diferentes instancias que caracterizan este espacio de actuación: Ia instancia de proyecto, la instancia legal y la instancia de aplicación del proyecto. La ausencia de alguno de ellos, aunque sea parcial, debilita las probabilidades de alcanzar los objetivos fijados y limita el rol protagónico del Estado como garante.

Con respecto al marco normativo en estudios previos (Erbiti, 2011; Barreto, 2015; Romagnoli, 2018; Seluy y Romagnoli, 2018, entre otros) se han realizado señalamientos referidos al ámbito regulatorio vigente en Argentina en materia de ordenamiento ambiental del territorio, en los cuales se reconoce el quiebre entre cuerpo normativo y aplicación eficaz de instrumentos legales.

Todos los aportes señalados constituyen parte del análisis de los antecedentes de esta investigación, cuya lectura metodológica promovió instancias de debate, que dieron origen al planteo del problema y permitieron su encuadre y recorte inicial.

\section{CONCLUSIONES Y REFLEXIONES PROUISORIAS}

La intención de trabajar en el campo de la planificación territorial, y específicamente en el ámbito de los instrumentos de la política, orientados al ordenamiento ambiental del territorio, tiene nuestro interés puesto en abordar aspectos de la gestión aún no suficientemente explorados, con el objeto de producir efectos en diferentes espacios de inserción.

En relación con el ámbito de las producciones científicas, el impacto que se espera se funda en el enfoque crítico que aquí se asume, pues se parte de una visión que trasciende e espacio circunscripto exclusivamente a las políticas de ordenamiento ambiental del territorio, y se plantea su articulación con otras dimensiones de análisis que suponen la valoración de los intereses individuales y colectivos, públicos y privados que entran en juego. Ello implica, por un lado, una mirada epistemológica, que presume partir de un abordaje que pretende superar los enfoques tecnocráticos. En tal sentido, se asume la perspectiva de la complejidad como proceso que se va configurando en distintas fases o momentos (politización de una necesidad/demanda, interpretación de esas necesidades, definición de satisfactores "adecuados" e implementación de acciones concretas), como producto de la disputa entre los diversos actores interesados e involucrados en las distintas instancias implicadas en su configuración (Subirats, 1989; Tamayo Sáez, 1997; Jobert, 2005; Bourdieu, 2001 y Romagnoli, 2015 y 2017) y como instrumento del Estado para la conformación, regulación y legitimación de grupos sociales.

Asimismo, se sostiene que el carácter complejo del objeto de estudio, de naturaleza epistemológica multidimensional, y que por lo mismo requiere "concurrencia interdisciplinaria" (Kullock, David; 2019), exige un diseño metodológico que pretende constituirse en el aporte de una estrategia que posibilite nuevas formas de analizar las políticas de ordenamiento ambiental del territorio.

En relación con los efectos sobre la actividad de formación universitaria, el proyecto prevé desde su inicio la conformación de espacios de intercambio en los que se planteen 
debates acerca del objeto de estudio, las problemáticas en que derivan las acciones sobre el territorio y las posibles estrategias metodológicas empleadas en su abordaje. En instancias del desarrollo de estas actividades se aspira a la identificación y formulación de problemas relativos a las políticas de ordenamiento ambiental del territorio, y a la construcción de una estrategia metodológica para el desarrollo de proyectos de investigación relativos al campo. Pero además, se espera que los resultados obtenidos aporten a la construcción del corpus teórico de la Maestría en Gestión Ambiental del Territorio Urbano de la Facultad de Arquitectura y Urbanismo, sede de esta investigación. En este mismo sentido, y en respuesta al cumplimiento de uno de los objetivos centrales de la propuesta, se pretende que ello constituya un aporte a la articulación de la MGATU y la FAU con otras unidades académicas de la UNNE, para concretar así instancias de vinculación entre actividades de investigación y posgrado, tal y como proponen los lineamientos de inserción de la propia universidad en el medio.

Como efecto secundario, aunque no menos importante, se espera contribuir también al desarrollo de las tesis de los maestrandos que integran el proyecto, y también del resto de los alumnos de la maestría, entendiendo que el objeto de estudio de esta investigación resulta fundamental para el abordaje de cualquier problemática vinculada con la gestión ambiental del territorio.

En relación con los efectos esperados sobre el medio local y regional (como posibles impactos del proyecto), la investigación en curso parte del supuesto de que el marco normativo de la política de ordenamiento ambiental del territorio en la Argentina, y específicamente en las provincias del NEA, presenta problemas vinculados con la fragmentación, superposición y el vacío normativo, lo cual se traduce en políticas de reducida incidencia en la gestión. Se sostiene que el análisis de los instrumentos legales vigentes en materia de ordenamiento ambiental del territorio puede constituirse en un aporte significativo para la construcción de un marco normativo unificado, que permita su integración efectiva al sistema de planificación. En tal instancia la formulación de lineamientos y recomendaciones - como parte de los resultados esperados del proyectopodrá ser transferida a organismos del Estado (de los distintos niveles jurisdiccionales involucrados) vinculados con políticas de ordenamiento ambiental del territorio, con el objeto de disponer de estrategias y contar con herramientas que posibiliten promover mejores condiciones de ocupación del territorio, aspirando a un mayor equilibrio socioeconómico, protegiendo y preservando el ambiente para condiciones más justas de desarrollo, y por lo mismo, creando las condiciones para acceder a mejores condiciones de vida urbana.

\section{REFERENCIAS \\ BIBLIOGRÁFICAS}

BARRETO, Miguel A.; ALCALÁ, Laura I.; BENÍTEZ, María A.; FERNÁNDEZ, María E.; PELLI, María B.; GIRÓ, M.; FERNÁNDEZ, María E. y ROMAGNOLI, Venettia. Colaboración especial: DEPETTRIS, Noel (2014). La política Federal de Vivienda desde su implementación en el Gran Resistencia (2003-2007). Análisis y recomendaciones. Buenos Aires: Diseño. BARRETO, Miguel; EBEL, Gabriela y ABILDGAARD, Evelyn (2015). Procesos recientes de ordenación y planificación territorial en Argentina y el nordeste. Actas digitales Encuentro XVI ENANPUR. Associação Nacional de Pós-graduação e Pesquisa em Planejamento Urbano e Regional. BOURDIEU, Pierre (1993). Esprits d'Etat. Revista Actes de la Recherche N. ${ }^{\circ}$ 96/97

BOURDIEU, Pierre (1998). La Dis- tinción. Criterio y bases sociales del gusto. Madrid: Taurus Ediciones. BOURDIEU, Pierre (2001). Las estructuras sociales de la economía. Buenos Aires: Manantial.

DOYAL, Len y GOUGH, Ian (1994). A theory of human need. London: Macmillan.

ESPING ANDERESEN, Costa (1989). Los tres mundos del Estado de Bienestar. Madrid: Alianza.

FRASER, Nancy (1986). Movimientos sociales vs. burocracias disciplinarias: los discursos de las necesidades sociales. Diánoia, Vol. 32, N. ${ }^{\circ}$ 32, 1986, pp. 167-202.

FRASER, Nancy (1991). La lucha por las necesidades: esbozo de una teoría crítica socialista-feminista de la cultura política del capitalismo tardío. Revista Debate Feminista. Marzo, 1991.

GALARZA LUCICH, Luisa (2002). Descentralización, organización económica del territorio y potencial de recursos. Lima: PNUD. Cuadernos PNUD, Serie Desarrollo Humano, 3. JOBERT, Bruno (2005). Estado, Sociedad, Políticas Públicas. Santiago de Chile: LOM Editores.

KULLOCK, David (2010). Planificación urbana y gestión social. Reconstruyendo paradigmas para la actuación profesional. Cuaderno Urbano (9), Buenos Aires: Nobuko-Eudene. pp. 243-274. ROMAGNOLI, Venettia (2012). La configuración de las necesidades en las políticas habitacionales a partir del caso del AMGR (Chaco). Avances en la construcción de un enfoque teórico para su abordaje. En Leoni, María S.; Leconte, Mariana y Sudar Klappenblach, Luciana (comp.) Libro de Actas. 446. XXXII Encuentro de Geohistoria Regional. Resistencia: Instituto de Investigaciones Geohistóricas, CONICET- UNNE, pp. 799-810

ROMAGNOLI, Venettia (2015a) El proceso de construcción de las necesidades en las políticas habitacionales y su incidencia en la configuración del territorio a partir del caso del Barrio Chelilly de Resistencia (Chaco, Argentina). 
En Carpio, María B.; Fantín, María A.; Leoni, María S. y Salinas, María L. (Comps.). Libro de Actas del XXXIV Encuentro de Geohistoria Regional. Resistencia: Instituto de Investigaciones Geohistóricas, CONICET- UNNE. pp. 547-558

ROMAGNOLI, Venettia (2015b).La disputa por la configuración de las necesidades habitacionales en las políticas públicas. Construcción de un marco interpretativo para su abordaje. ADNea Arquitectura y Diseño del Nordeste Argentino, Vol. 3, N. ${ }^{\circ}$ 3. Editorial de la Facultad de Arquitectura y Urbanismo de la UNNE. Pp. 101-118.

ROMAGNOLI, Venettia (2015). ¿Qué vivienda necesitan los pobres? Un estudio sobre la configuración de las necesidades en las políticas habitacionales vigentes a partir del caso del Área Metropolitana Gran Resistencia, AMGR (Chaco, Argentina). Tesis doctoral inédita. Pp. 448. ROMAGNOLI, Venettia (2017a). La Política Federal de Vivienda en el AMGR (Chaco, Argentina). Disputas, interpretaciones hegemónicas y expresiones territoriales del proceso de configuración de las necesidades habitacionales en las políticas públicas. En Revista ADNea Arquitectura y Diseño del Nordeste Argentino. Vol. 5 N. ${ }^{\circ}$ 5. Editorial de la Facultad de Arquitectura y Urbanismo de la UNNE. Pp. 97-106.

ROMAGNOLI, Venettia (2017 b). El proceso de construcción de las necesidades en las políticas habitacionales. Un modelo teórico-metodológico para su análisis desde una perspectiva interpretativa. Revista Perspectivas de Políticas Públicas, Vol. 7 N. ${ }^{0}$ 13. Departamento de Planificación y Políticas Públicas UNLa. Pp. 89-107.

SUBIRATS, Joan (2001). El análisis de las políticas públicas. GAC SAINT No 15, Vol. 3, pp. 259-264.

TAMAYO SÁEZ, Manuel (1997.) El análisis de las políticas públicas. En: Bañón, Rafael y Carrillo, Ernesto (comp.). La nueva administración pública. Madrid: Alianza Universidad. pp. 2-22. 